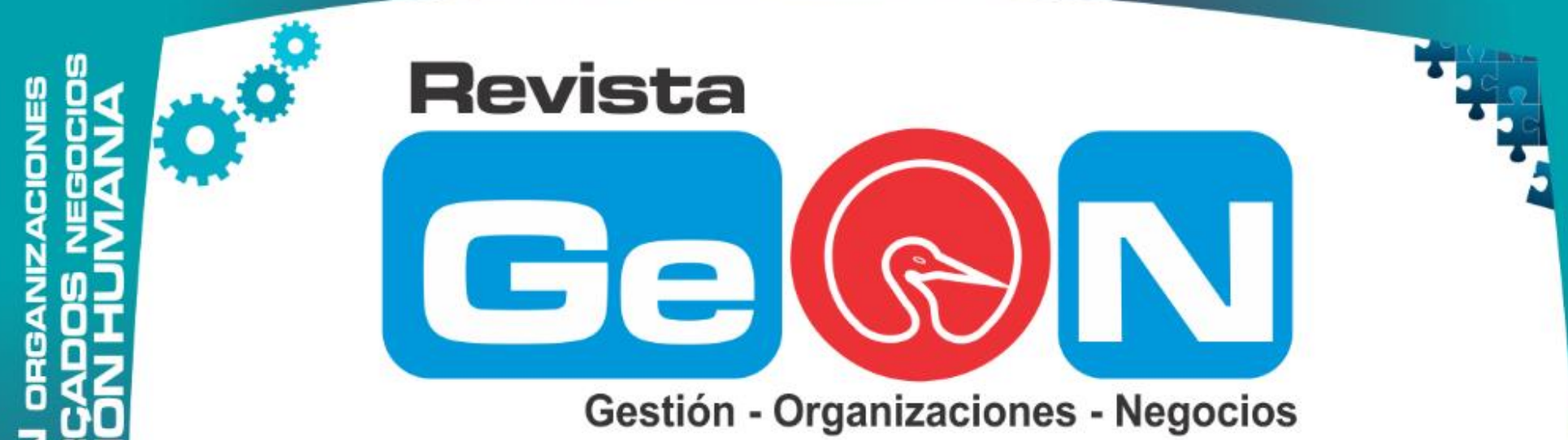

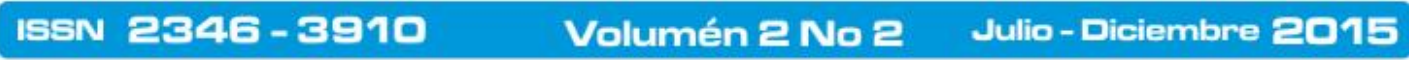

Revista Electrónica de la Facultad de Ciencias Económicas de la Universidad de los Llanos

1. 3 II

440

VILLAVICENCIO - COLOMBIA

$>2$

64

2

2 平品

70

ব)

展

o

ii III

0 U

ใด

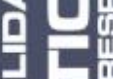

居的

임

Iiin in

照

只

造

iil 2

if in

4 吕

2

400

付 iI
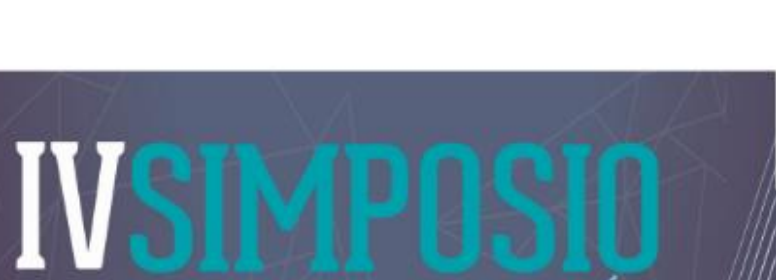

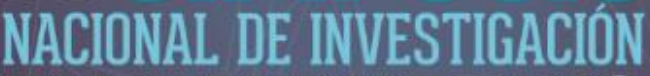

EN MARKETING
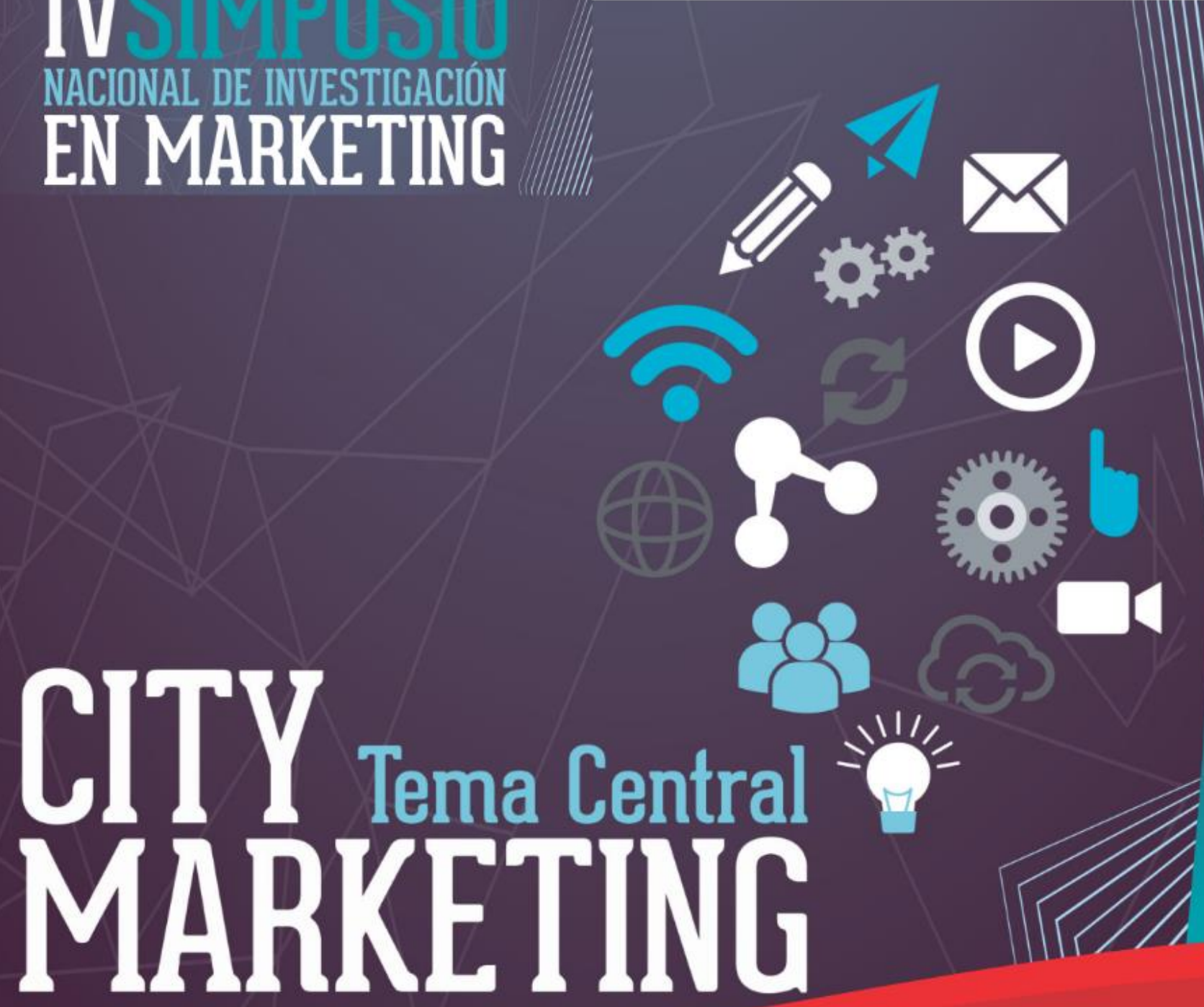

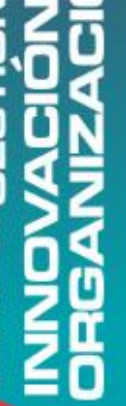
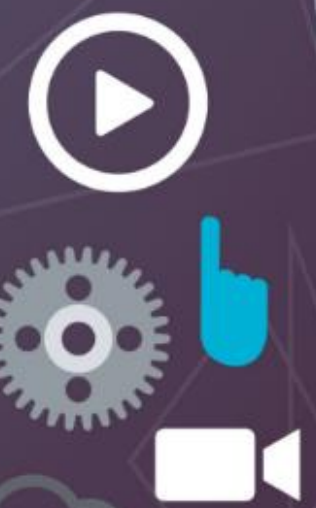


\section{Reacciones de los consumidores en el polígrafo ante una propaganda sobre medio ambiente - María Orquídea Escobar}

\author{
María Orquídea Escobar Torres \\ Magister en Dirección de Marketing \\ orquideaescobar@gmail.com.
}

\section{Resumen}

En mercadeo, resulta difícil conocer las reacciones de los consumidores ante los estímulos publicitarios. Estas reacciones con frecuencia no se pueden observar de manera directa, por lo cual existe una tendencia a emplear tecnología especializada para medir el comportamiento de los individuos ante diferentes estímulos. En esta investigación se busca conocer las respuestas psicofisiológicas ante una propaganda sobre medio ambiente, medidas por medio del polígrafo, en 20 personas, hombres y mujeres estudiantes universitarios y profesionales, con edades entre 18 y 48 años, en Bogotá. Los resultados muestran una diferencia en cuanto a la respiración torácica y abdominal y evidencian contradicciones entre el reporte verbal sobre el impacto de la propaganda y las reacciones frente al polígrafo.

\section{Palabras clave}

Polígrafo; Marketing; Comportamiento del consumidor; Propaganda; Neuromarketing.

\footnotetext{
Abstract

In marketing, it is difficult to know the reactions of consumers to advertising stimuli. These reactions often cannot be observed directly, so there is a tendency to employ specialized technology to measure the behavior of individuals to different stimuli. This research seeks to understand the psychophysiological reactions on environmental advertising in through the polygraph, on 20 people, male and female university students and professionals, aged 18 and 48, in Bogota. The results show a
}

difference in the thoracic and abdominal breathing and evident contradictions between verbal report on the impact of advertising and reactions on the polygraph.

\section{Keywords}

Polygraph; Marketing; Consumer behavior; Advertising; Neuromarketing.

\section{Introducción}

En el estudio del comportamiento del consumidor, no siempre es fácil establecer el impacto que tendrá una acción publicitaria, especialmente cuando partimos del análisis de sus reportes verbales; pues con frecuencia, las personas manifiestan una posición respecto a un producto o servicio determinado, lo que no siempre está en concordancia con su comportamiento de compra y uso de bienes y servicios.

Con el interés de explorar en profundidad las verdaderas reacciones del consumidor ante productos o estímulos publicitarios, el neuromarketing intenta indagar sobre el efecto que estos tienen en el cerebro, como una forma de conocer reacciones autónomas que se dan independientes de la voluntad de los individuos.

Una manera de averiguar este tipo de reacciones ante la publicidad se encuentra en el polígrafo, el cual permite medir respuestas psicofisiológicas autonómicas, que se derivan de la captación de estímulos de diferente tipo (American Polygraph Association, 2010).

Durante los años 2013 y 2014 se desarrolló en la Corporación Universitaria UNITEC en Bogotá, el proyecto de investigación denominado Relación entre perfiles de personalidad y tipo de respuestas psicofisiológicas ante un estímulo publicitario, según género. A partir de la revisión teórica 
sobre el tema se llevó a cabo el trabajo de campo, que incluyó pruebas de polígrafo y aplicación del Cuestionario 16PF para medir la personalidad.

En esta ponencia, se presentan los objetivos, aspectos metodológicos y principales resultados de la investigación en su fase experimental con las pruebas de polígrafo, sin contemplar la relación con las variables perfil de personalidad y género. Se incluyen las conclusiones correspondientes y algunas ideas respecto a la investigación que da continuación a este proyecto.

\section{Aspectos metodológicos}

El estudio realizado tuvo como objetivo, conocer las respuestas psicofisiológicas ante una propaganda sobre medio ambiente, medidas por medio del polígrafo, entre estudiantes y profesionales en Bogotá. Las mediciones se realizaron sobre tres indicadores registrados mediante este instrumento: Actividad Electro Dermal (EDA), alteración psicofisiológica del ritmo cardiaco y la presión sanguínea, y cambios en la respiración torácica y abdominal.

La investigación fue de tipo experimental correlacional; pero como se mencionó, en esta ponencia se hará referencia únicamente a la fase experimental, sin tomar en cuenta la correlación con otras variables. Recordemos que un estudio experimental es aquel, "en el que se manipulan intencionalmente una o más variables independientes (supuestas causas-antecedentes), para analizar las consecuencias que la manipulación tiene sobre una o más variables dependientes (supuestos efectos-consecuencias), dentro de una situación de control para el investigador" (Hernández Sampieri, 2003).

En esta investigación, la variable independiente fue la pieza de propaganda denominada "Medio ambiente", la cual se presentó a los participantes. El efecto o variable dependiente, estuvo representado en las respuestas psicofisiológicas medidas por medio del polígrafo.
La muestra estuvo conformada por 20 participantes, 14 mujeres y 6 hombres, con edades entre 18 y 48 años, quienes participaron en el estudio según su disponibilidad. De los participantes, 16 eran estudiantes universitarios y 4 profesionales pertenecientes a carreras o programas no relacionados directamente con publicidad, mercadeo y cine; esta condición se estableció para evitar el sesgo en la apreciación de los estímulos publicitarios, derivado de la profesión con la que estaban más relacionados. Los participantes eran afines a: administración, finanzas, ingeniería, derecho, psicología e investigación criminal, entre otras.

En la preparación del material de prueba, inicialmente se evaluó la respuesta en el polígrafo, de tres piezas publicitarias y una de propaganda, a saber:

"The Force": Volkswagen Commercial (https://www.youtube.com/watch?v=R55euHQna0)

"Ghost car" (https://www.youtube.com/watch?v=Wz1W om igwg)

"For Warriors" Nike Commercial (https://www.youtube.com/watch?v=O4FXx6kh $\underline{\mathrm{WtI}})$

"Medio ambiente" (https://www.youtube.com/watch?v=zlzTQUjS fk).

Dentro de estas cuatro piezas, se observó que la propaganda, llamada "Medio ambiente", que muestra el suicidio de tres animales al observar el deterioro de nuestro planeta, generaba mayor reacción emocional en el observador, según se reflejaba en los registros del polígrafo.

Antes de llevar a cabo las pruebas experimentales, se efectuaron experiencias piloto con el polígrafo, para ajustar el procedimiento en la realización de las pruebas. Todos los registros que aporta el polígrafo fueron tomados en la muestra de veinte (20) voluntarios del estudio a quienes se les presentó como estímulo la 
propaganda "Medio Ambiente". Mientras los sujetos estaban conectados al equipo, se realizó el siguiente procedimiento que involucra algunas preguntas específicas:

C1- ¿Está sentado (a) en este momento?- Es una pregunta comparativa efectuada antes de ver el video, que permite establecer las reacciones fisiológicas normales del sujeto analizado.

R1- Presentación del video: Propaganda "Medio ambiente"- (Estímulo con 56 segundos de duración).

C3- ¿Hoy es sábado?- Pregunta posterior al video, que permite volver a la situación normal al sujeto examinado.

R4- ¿Te impactó este video?- Pregunta relevante, posterior al vídeo, que ante la respuesta cerrada Si/No, permite medir la veracidad o no veracidad psicofisiológica de esa respuesta.

C5- ¿La luz está prendida? - Pregunta comparativa posterior al video, que permite volver de nuevo la normal fisiológica del examinado.

Para efectuar la prueba de polígrafo, se citaron a los participantes a una sala adecuada para el fin del estudio, donde se tenía instalado el polígrafo con los diferentes elementos para efectuar la medición y un televisor donde se veía y escuchaba la propaganda escogida para las pruebas. Los sujetos fueron informados previamente sobre el procedimiento de medición con el polígrafo y la prueba de personalidad, recibiendo la aceptación y la participación voluntaria de cada uno de ellos. El psicólogo experto en la aplicación del polígrafo realizó a cada participante una corta entrevista y la prueba de polígrafo, tomando registro de las expresiones verbales de los participantes en el estudio, antes, durante y después de la aplicación de la prueba de polígrafo mientras se presentaba la propaganda.

Como instrumento principal se empleó el polígrafo. Según la APA - American Polygraph
Association (2010), el término "polígrafo" significa literalmente "muchos escritos". El nombre se refiere a la manera en que las actividades fisiológicas seleccionadas se registran simultáneamente.

Según la información suministrada por el psicólogo poligrafista, Doctor Valbuena, B. F. (2014), quien participó como asesor metodológico de este estudio, se utilizó un equipo de polígrafo digital (computarizado) marca Lafayette, referencia LX 4000; debe aclararse que se trata del mejor y más popular polígrafo computarizado, el cual es capaz de analizar y grabar de forma simultánea, múltiples variables fisiológicas, mientras el entrevistado responde una serie de preguntas en un cuestionario estructurado y específico para el tema de investigación

Explicó el Dr. Valbuena, cómo las gráficas generadas durante el examen fueron luego interpretadas por el examinador utilizando para ello las múltiples herramientas de software que la Suite del LX trae incluida. Los resultados de la grabación fueron arrojados por los parámetros normales, aprobados y científicamente sustentados de la poligrafía: Respiración abdominal y torácica, (EDA), pulso y presión sanguínea. Para efectuar dichas mediciones, el polígrafo cuenta con los siguientes sensores o aditamentos: 1) Neumógrafo: Encargado de medir y registrar la actividad respiratoria (inspiración y expiración) del individuo; el método para aplicar éste, dice el Dr. Valbuena, consiste en sujetar dos tubos (neumo), uno en la parte del tórax y el otro en el abdomen, la función de estos es registrar el movimiento torácico. 2) Galvanómetro: Su función es medir y registrar por medio de sensores los cambios que se presentan por la transpiraciónelectricidad, EDA; el método para aplicar, es sujetar los Galvanómetros en los dedos corazón e índice. 3) Cardiógrafo: El empleo del Baunómetro es para medir y registrar el movimiento del corazón, la presión sanguínea y el ritmo del pulso del individuo; el método para aplicarlo, es sujetar el brazalete de cardiología en uno de los brazos del sujeto. 
Los registros se hacen sobre el sistema nervioso autónomo- SNA- que recibe la información de las vísceras y del medio interno, para actuar sobre sus músculos, glándulas y vasos sanguíneos; es un sistema involuntario y transmite impulsos desde el sistema nervioso central hasta la periferia estimulando los aparatos y sistemas órganos periféricos. Controla la frecuencia cardíaca y la fuerza de contracción, la contracción y dilatación de vasos sanguíneos, la contracción y relajación del músculo liso en varios órganos, acomodación visual, tamaño pupilar y secreción de glándulas exocrinas y endocrinas, regulando funciones tan importantes como la digestión, circulación sanguínea, respiración y metabolismo.

Durante la prueba se obtuvo un registro de las respuestas psicofisiológicas de los participantes, impreso por el polígrafo, en lo relativo a: La reacción psicofisiológica denominada Actividad Electro Dermal (EDA), ante la propaganda; la alteración psicofisiológica del ritmo cardiaco y la presión sanguínea; y la reacción psicofisiológica en la respiración torácica y abdominal. Los registros de las reacciones psicofisiológicas ante el polígrafo, se convirtieron a un lenguaje numérico.

Posteriormente se llevó a cabo el procesamiento estadístico mediante el programa SPSS, con la orientación del psicólogo Octavio Escobar (2014), para obtener los resultados del polígrafo, de tal forma que se pudieran interpretar para responder a los objetivos planteados en este estudio.

\section{Resultados}

En la Figura 2, se presentan las reacciones psicofisiológicas del grupo de individuos medidas en diferencias entre dos momentos de la aplicación: a) Antes de presentar la propaganda y b) durante la presentación de la propaganda. Así mismo, se evalúan tres aspectos que mide el polígrafo: Respiración, EDA y tasa cardiaca. Se puede apreciar el peso de las distintas reacciones, encontrándose que el indicador que presenta diferencias más marcadas es la respiración, con una correlación de 0,755. En una proporción mucho más baja aparecen la diferencia en EDA 0,285 y la relativa a los cambios en la tasa cardiaca 0,243.

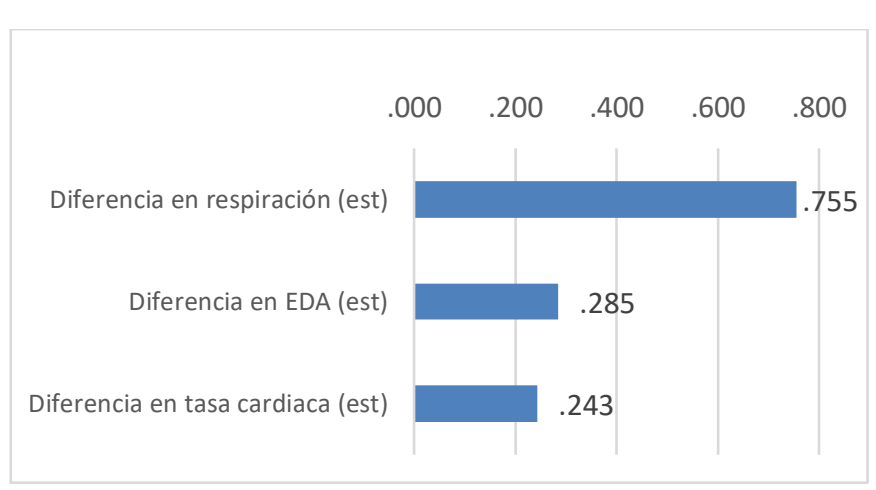

Figura 2. Diferencia entre dos momentos en la aplicación de la prueba de polígrafo. Fuente de elaboración propia

\section{Contradicciones de los participantes}

Al analizar los resultados de las respuestas psicofisiológicas y los reportes verbales de los participantes, se encontró un aspecto muy interesante, relacionado con las contradicciones que muestran los sujetos al expresar sus percepciones sobre el impacto que les produjo el estímulo específico observado ("medio ambiente") y la reacción de cada sujeto registrada en el polígrafo durante la presentación del video.

Así se pudo observar, como entre los individuos que respondieron verbalmente de manera afirmativa a la pregunta relacionada con el impacto de la propaganda, la respuesta psicofisiológica registrada en el polígrafo se mostraba contraria a dicha afirmación.

De manera similar, algunos de los entrevistados expresaron un bajo impacto de la propaganda, mientras que el registro del polígrafo evidenciaba reacciones psicofisiológicas inversas. En la Figura 3 se aprecia un registro que muestra alto impacto en un sujeto que expresa lo contrario.

\section{Conclusiones}

Como se enunció al comienzo, el estudio realizado buscaba conocer las reacciones 
psicofisiológicas que se presentaban en un grupo de individuos, ante la exposición de la propaganda denominada "Medio Ambiente".

En la parte inicial del experimento, según lo planeado, se encontró una mayor diferencia en la reacción ante el polígrafo antes de y durante la presentación de la propaganda, en lo relativo a la respiración torácica y abdominal, respecto a los cambios en el ritmo cardiaco y la Actividad Electro Dermal - EDA.

Sin embargo, el hallazgo que más llama la atención es la contradicción encontrada entre las respuestas dadas a la pregunta sobre el impacto de la propaganda y el registro observado en el polígrafo. Esto llevó a inquietarse sobre lo que podría llamarse la conducta de mentir o el engaño de los consumidores. Veamos algunos análisis al respecto.

Aunque con frecuencia, el indicador más confiable en la medida de engaño es el EDA, en este estudio la correlación más marcada la tienen los valores de la respiración, teniendo en cuenta que es la medida de mayor rango o longitud, lo cual la hace más prominente. Igualmente, esto obedece a que los movimientos del tórax y el abdomen que se realizan en el momento de un cambio en la respiración, son más evidentes que en los otros dos indicadores.

Teniendo en cuenta que la pieza presentada tiene un componente visual más marcado mostrando imágenes que podrían considerarse impactantes, podría suponerse que los estímulos de carácter más emotivo tendrían un elemento que alterara sensiblemente la respuesta del organismo. Sin embargo, esta afirmación se contradice con el análisis de las respuestas suministradas por los participantes, en tanto, varios de ellos manifestaron que la propaganda les resultaba impactante estando en contravía de lo que reflejaba el registro del polígrafo.

De manera similar, sucedió con otros individuos quienes expresaron un bajo impacto de la propaganda, estando en contradicción con lo que mostraba el polígrafo. Lo anterior pone en evidencia, un comportamiento muy frecuente en nuestro medio, relacionado con lo que podría denominarse la "conducta de mentir", la cual ha sido objeto de estudio por varios autores interesados en el tema del neuromarketing y en áreas afines.

Podemos citar a García Martínez (2013), quien en su artículo La gran brecha entre lo que dicen y realmente piensan los consumidores, hace referencia al estudio "Secretos y mentiras" realizado por Young \& Rubicam en Estados Unidos, Brasil y China; la cual expresa "que los consumidores mantienen en realidad puntos de vista opuestos a lo que dicen". Un aspecto del estudio hace referencia a que los sujetos expresaban, que lo más importante para ellos consistía en dar sentido a su vida; mientras que en sus motivaciones inconscientes, se podía apreciar la alta puntuación que ellos le otorgaban a la satisfacción sexual; esta contradicción podría estar basada en la incomodidad que representa para la gente admitir lo estimulante que le resulta el sexo. Otros autores como Lindstrom (2009) y Graves (2011), reportan consecuencias lamentables en casos donde el consumidor ha presentado la conducta de engañar, intentando mostrar una imagen diferente a la real, por conveniencia o simplemente debido a motivos inconscientes que no puede realmente explicar.

Como continuación a esta investigación, en UNITEC se está desarrollando un estudio de revisión teórica respecto de las bases conceptuales y los estudios recientes relativos al comportamiento de mentir en consumidores, con el objeto de aportar explicaciones a este fenómeno y construir una base de conocimiento que permita efectuar en un futuro cercano, estudios experimentales sobre las contradicciones de las personas en sus acciones de compra y consumo respecto a sus reacciones psicofisiológicas o cerebrales.

Esto permitirá entender mejor este comportamiento del consumidor y detectar sus verdaderas motivaciones y reacciones, para en consecuencia, tomar decisiones mejor orientadas y efectivas. 


\section{Referencias}

American Polygraph Association - APA (2010). Sitio web de APA, [en línea], disponible en: http://www.polygraph.org/section/researchstandards-apa-publications.

Cortés, A. (2006). "Reflexiones sobre el papel de la publicidad institucional en la educación social". Sevilla-Cádiz: Centro Andaluz de Estudios Empresariales (CEADE, Sevilla).Grupo Comunicación y Poder (Universidad de Málaga). España. ac@alfonsocortes.com Grupo de Trabajo: Publicidad y marketing. IX Congreso IBERCOM.

Escobar, O. (2014). Entrevista personal octavioescobar@gmail.com

García Martínez, M.A. (2013). "La gran brecha entre lo que dicen y realmente piensan los consumidores", sitio web de Puro Marketing, [en línea], disponible en: http://www.puromarketing.com/88/18083/granbrecha-entre-dicen-realmente-piensan- consumidores.html [accesado el día 21 de noviembre de 2014]

Graves, P. (2011) “¿Por qué consumimos? El mito de los estudios de mercado, y las verdades sobre la psicología del comprador y su comportamiento". Barcelona: Ediciones Urano.

Lindstrom, M. (2009). "Compradicción. Verdades y mentiras de por qué las personas compran". Bogotá: Editorial Norma.

Rodríguez, D. A. (2008). "El carácter como determinante diferencial para el posicionamiento de las marcas". Tesis de grado Facultad de Comunicación, Pontificia Universidad Javeriana, Bogotá.

Valbuena, B. F. (2014). Sitio web de la Universidad Javeriana, [en línea], disponible en:

http://www.javeriana.edu.co/biblos/tesis/comuni cacion/tesis48.pdf

Entrevista personal. belfer71@gmail.com 